\title{
Les élites francophones du collège des Frères de Jérusalem, 1922-1939
}

Entre instrumentalisation politique, construction identitaire et usages religieux

Karène Sanchez-Summerer

\section{OpenEdition}

Journals

\section{Édition électronique}

URL : https://journals.openedition.org/dhfles/146

DOI : $10.4000 /$ dhfles. 146

ISSN : 2221-4038

Éditeur

Société Internationale pour l'Histoire du Français Langue Étrangère ou Seconde

\section{Édition imprimée}

Date de publication : 1 janvier 2007

Pagination : 107-129

ISSN : 0992-7654

Référence électronique

Karène Sanchez-Summerer, «Les élites francophones du collège des Frères de Jérusalem,

1922-1939 ", Documents pour l'histoire du français langue étrangère ou seconde [En ligne], 38/39 | 2007, mis en ligne le 16 décembre 2010, consulté le 27 mai 2021. URL : http://journals.openedition.org/ dhfles/146 ; DOI : https://doi.org/10.4000/dhfles.146

Ce document a été généré automatiquement le 27 mai 2021

(c) SIHFLES 


\title{
Les élites francophones du collège des Frères de Jérusalem, 1922-1939
}

Entre instrumentalisation politique, construction identitaire et usages religieux

\author{
Karène Sanchez-Summerer
}

"L'oreille de l'oriental est habituée aux sons
étrangers "1. Père Dhorme, prieur de l'École
biblique et archéologique de Jérusalem, 1922.

L'Angleterre n'était à peu près rien à Jérusalem, elle n'y avait pas de fonction de quelque importance, sa langue n'y était pas parlée, tandis que les œuvres les plus florissantes étaient françaises et qu'elles avaient répandu la langue française autant et plus même qu'en aucun coin de cet Orient que domine notre influence intellectuelle. [...] C'est sur la conservation des établissements des religieux français et $\mathrm{du}$ caractère résolument français de leur enseignement que nous devons concentrer nos efforts. C'est là qu'est le réduit de notre influence. (Robert de Caix au ministre des Affaires étrangères, La position de la France en Palestine, 28 octobre 1920, AMAE, E 312/1, n 404)

1 Pour la communauté catholique française, au début des années 1920, tandis que s'est pratiquement jouée la perte du protectorat catholique français en Terre sainte face aux Britanniques, la Palestine demeure « la plus française des terres d'Orient $»^{2}$.

2 Le collège des Frères de Jérusalem, fondé en 1876, constitue un cas exceptionnel (de par sa position au cœur du quartier chrétien de la vieille ville et de par son écho auprès des différentes communautés ethniques et religieuses de la ville) de collège d'enseignement primaire, secondaire et professionnel, représentatif du statut du français comme langue seconde des élites cultivées ${ }^{3}$. Représentatif, l'établissement des frères l'est dans la mesure où il nous renseigne sur le milieu d'origine de ses élèves et nous permet ainsi de brosser un portrait des élites palestiniennes. En amont, il nous permet de comprendre les politiques menées par les deux puissances mandataires de la région qui s'affrontent, et en aval, certains aspects de l'usage du français dans cette société, bien différente de ceux de Syrie et du Liban, sous mandat français. L'étude de certaines archives inédites permet de mettre en valeur un des aspects de cette politique linguistique française $e^{4}$. Un des buts affirmés du consulat général de France à Jérusalem 
au début de la période mandataire britannique reste d'ailleurs clairement de «former une élite $»^{5}$.

Les frères n'ouvrent au départ qu'une école gratuite en octobre 1878, puis en 1904, la section payante, le collège Saint-Jean Baptiste de La Salle, ouvre ses portes, conformément à la demande de certains parents qui occupent une position sociale, morale, politique et économique relativement importante dans la société de Jérusalem, qui exercent une influence efficace sur les prises de décisions et bénéficient d'un prestige social ${ }^{6}$. Les frais d'inscription représentent à peu près cinq fois le salaire mensuel d'un ouvrier ${ }^{7}$. Un large appel est lancé au français, comme instrument de culture selon une double logique: chez les non-musulmans, comme langue de protection et d'éducation, chez les musulmans, le français était jusqu'en 1918 la deuxième langue d'administration de l'empire, et sa connaissance était indispensable pour faire carrière à l'intérieur du système ottoman rénové 8 .

Diverses temporalités peuvent être considérées durant cette période : temps court du passage d'un pouvoir à l'autre (un mandat de vingt-six ans, débutant en 1922, après quatre ans de présence militaire, face à une influence française séculaire), temps long de certaines réactions britanniques au niveau éducatif (arsenal législatif mis en place dans les années 1930), avec de profondes conséquences pour la société palestinienne. La perception du temps par les frères est différente de celle des autorités. Les frères prennent en effet conscience de la nécessité de s'adapter et de sauvegarder l'enseignement et la pratique du français tout en favorisant l'anglais pour des raisons de débouchés professionnels pour leurs élèves.

5 Ainsi il est intéressant d'observer à travers cette étude de cas, qui s'inscrit dans la compréhension de la politique française au Levant ${ }^{9}$, le partage progressif entre le français et l'anglais en tant que langue des élites, les différentes tentatives effectuées pour le maintien de la langue française comme vecteur de l'influence française, les modalités selon lesquelles cette mutation s'opère au sein du collège et au-delà, saisir ce portrait évolutif des élites hiérosolymitaines ${ }^{10}$. 


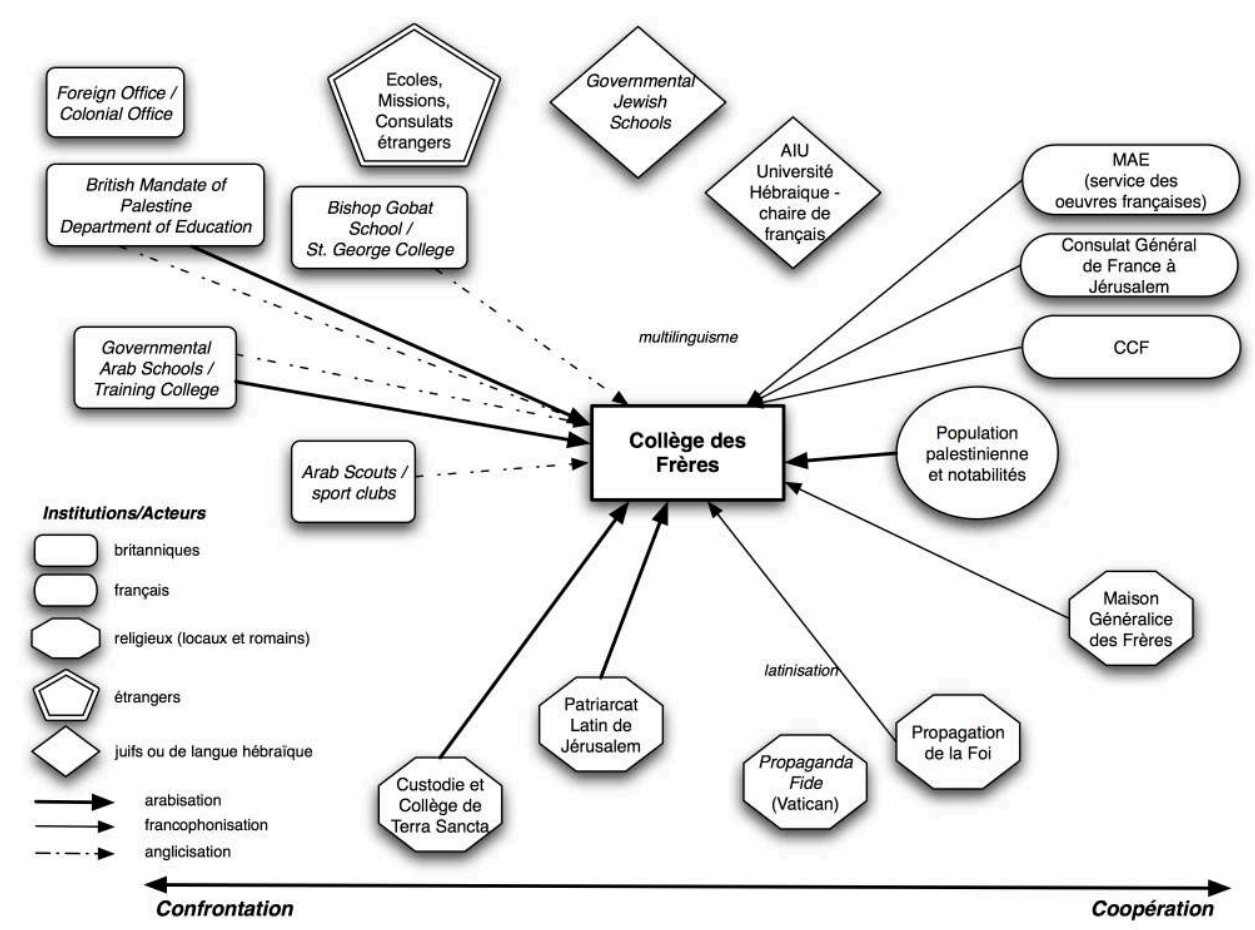

\section{Entre instrumentalisation politique et patriotisme, un collège « vecteur » de l'influence française}

« Une Babel linguistique sur fond d'arabe et de français dominant. »Catherine Nicault ${ }^{12}$

$6 \mathrm{Au}$ cours de cette période, deux sphères d'influence, anglophone et francophone, s'affrontent. Pour les frères se pose alors la question de savoir comment rester loyaux envers la France tout en étant sommés par la puissance mandataire de poursuivre une reconnaissance des pouvoirs anglais et ses implications dans le domaine éducatif.

7 À la tête d'un collège de référence dès sa création et auréolé par les élites locales et les pouvoirs français à la fin de l'empire ottoman ${ }^{13}$, les frères sont d'abord confiants, malgré leurs inquiétudes, dans les accords franco-anglais, qui promettaient d'assurer une autonomie réciproque des écoles, sous les deux mandats respectifs. Dès le début des années 1920 pourtant, les différents émissaires de la France comprennent qu'elle a perdu la bataille territoriale et son Protectorat, et se font l'écho des inquiétudes grandissantes des frères pour la préservation de leur institution.

\section{Affrontement entre deux sphères d'influence}

Depuis les années 1910, Jérusalem connaît un véritable bouillonnement de ses structures éducatives ${ }^{14}$, de tous bords, chacune revendique une forme de primauté. Les années 1930 voient aussi le développement intense du processus de scolarisation (enseignement public et privé), dû entre autres aux subventions accordées par les consulats étrangers, et à l'appui de la Grande-Bretagne aux écoles et collèges gouvernementaux et quelques institutions anglicanes ${ }^{15}$. 
Jérusalem est depuis longtemps la ville des églises et des couvents; on pourrait présentement l'appeler la ville des écoles car ardent est le zèle pour la formation de la jeunesse qui s'est réveillé pour ne pas dire enflammé chez toutes les nations et les confessions. Bientôt la ville ne sera qu'une immense université ${ }^{16}$.

Même si les lazaristes n'ont pas de collège à Jérusalem (ils sont présents et influents ailleurs dans le Levant, notamment à Damas), la concurrence est de taille pour le collège des frères.

Nombre d'écoles étrangères en Palestine (nombres d'écoles et d'élèves)(d'après le Annual Report of Education, Department of Education, Government of Palestine)

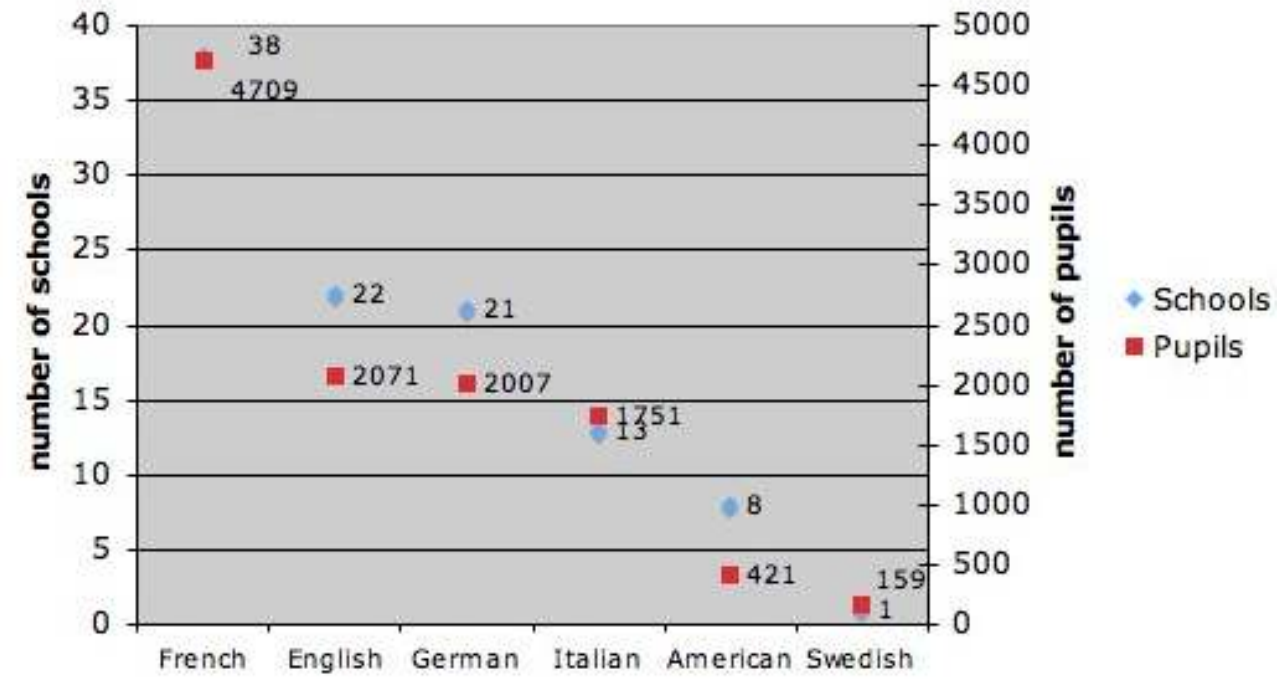

Face à la présence séculaire française, les Britanniques cherchent rapidement, mais progressivement, à asseoir leur position et leur action linguistique et culturelle, préparée par les entreprises antérieures ${ }^{17}$. Parallèlement à la délimitation des frontières du mandat français, le consulat général perçoit les difficultés dues aux nouvelles dispositions législatives notamment car, au-delà de la volonté affirmée par le Haut-commissariat britannique de préserver la liberté des œuvres françaises, les garanties formelles assurant le statu quo ne sont pas affirmées ${ }^{18}$.

Il est plus difficile de sauvegarder la liberté et le caractère nettement national de

l'action de nos œuvres sous le régime qui s'établira en Palestine ${ }^{19}$.

11 Tandis que pour les Britanniques « le Levantin gallicisé est représenté comme un être factice, qui réunit en lui tous les défauts des Français et des indigènes sans aucune de leurs qualités $»^{20}$, certaines élites de Jérusalem réclament leur rattachement à la Grande Syrie. Pour beaucoup d'élites, la présence des Britanniques n'est pas perçue comme étant de longue date, ni comme culturelle et religieuse.

Dès les premiers pas en Palestine, un fait s'impose avec évidence: les occupants d'aujourd'hui n'avaient hier encore, aucune racine dans ce pays, ils n'y avaient acquis aucune sympathie ${ }^{21}$.

Dès 1922, l'Education Department est créé au sein du Palestine Government. Les articles 15-16 du mandat insistent d'ailleurs sur le devoir éducatif des autorités envers les populations locales. En 1924, une nouvelle loi entraîne un refus catégorique de la part des frères, elle stipule que la langue française n'est plus une langue officielle aux examens $^{22}$. En 1933, l'Education Ordinance exige notamment des licences d'enseignement 
pour les professeurs, des inspections des agents de l'Education Department, et des heures d'enseignement d'anglais, avec des formulaires annuels à renvoyer au Hautcommissariat ${ }^{23}$. La direction du collège y voit une tentative d'ingérence de la part de la puissance mandataire, malgré les visites sans heurts des officiels au collège :

23 mars 1926, la maison reçoit la visite de Mr Ronald Storrs, gouverneur de Jérusalem, accompagné de Mr Gaston Maugras, Consul général de France. Son Excellence félicite les maîtres de la solide instruction et de la bonne éducation qu'ils donnent à la jeunesse hiérosolymitaine et encourage les jeunes à être fidèles aux bons principes qui leur sont inculqués ${ }^{24}$.

\section{Vecteur de l'influence culturelle française, «Éveiller en eux et fortifier le sentiment de la patrie »}

13 L'œuvre d'enseignement effectuée par les frères apparaît rapidement comme une plate-forme d'influence, voire, une " reconquête " pacifique par la langue ${ }^{25}$. Il s'agit de :

former des élites influentes qui soient favorables à la France. Il n'y a aucun intérêt à disséminer nos efforts et à soutenir de nombreuses écoles dans les villages [...] le français n'a jamais été la langue véhiculaire des gens du peuple. En ce pays, le français ne peut et ne doit être que la langue de l'élite, ce qu'il a d'ailleurs toujours été, même depuis l'occupation britannique. Je connais de hauts fonctionnaires britanniques arrivés en ce pays sans parler le français et qui l'ont appris ici de nos congrégations ${ }^{26}$.

14 À un moment où la France mène une bataille sur son sol métropolitain contre les frères, elle leur réitère son soutien à Jérusalem ${ }^{27}$, les conforte dans leur rôle éducatif, le collège étant pour le consulat général un lieu de "propagande civique », soutient la mission de la congrégation des frères. Le consul G. Maugras lui-même déclare d'ailleurs aux frères qu'il convient d'« éduquer de bons chrétiens qui connaissent la langue de leurs formateurs ». Dès 1919, le rapport du frère Justinus souligne leur double allégeance, qui se manifeste à travers " une politique d'expansion à l'étranger sous la double inspiration de la foi religieuse et du sentiment patriotique ${ }^{28}$. Cette forme de patriotisme ne va d'ailleurs pas sans inquiéter les autorités supérieures des frères ${ }^{29}$. En 1927, le prieur des dominicains, le $P$. Dhorme, déclare se féliciter « du triple lien Patrie Education Religion qui garantit la pérennité de l'union que tant de causes tendent à dissoudre $»^{30}$.

À partir des années 1930 cependant, face à la déconsidération institutionnalisée de la langue française de la part des Britanniques, et leur politique d'anglicisation qui tend à réduire le français à la part congrue, le consulat général insiste davantage sur l'image à véhiculer par les frères d'une France universaliste, moins chrétienne. « Après la religion, c'est la culture, la vraie, qui devient l'outil d'une pénétration française en Palestine ${ }^{31}$.

Le consulat général met en œuvre sa politique linguistique auprès des élites francophones de plusieurs manières. À côté des actions de soutien local à la «famille des francophones $»^{32}$, des récompenses nationales sont attribuées à l'institution par les pouvoirs publics. Le fondateur du collège est ainsi récompensé à l'Assemblée, et par l'Académie :

Le frère Evagre fut l'un des principaux artisans de ces merveilleux résultats d'expansion française, il s'agit aujourd'hui de ne pas laisser péricliter ces résultats, car l'usage du français dans nos écoles est menacé3 ${ }^{33}$.

17 Le soutien financier au collège est constant, mais de nature inégale ${ }^{34}$. Le consulat réitère ses demandes de subventions plus importantes au ministère des Affaires 
étrangères tout au long de la période. Ces demandes se justifient pour le consulat par le rôle éminent qu'elles endossent.

Les subventions de l'État français sont très faibles, parfois dérisoires. Ces établissements font pourtant une œuvre éminemment française, si notre influence se maintient en Palestine, c'est uniquement grâce à eux. Leur disparition serait désastreuse pour nous dans tout l'Orient ${ }^{35}$.

En 1930, les allocations accordées sont multipliées par trois. D'autre part, les projets d'extension des bâtiments des frères près des nouveaux quartiers bâtis, montrent leur désir de s'adapter à la demande au niveau spatial. Enfin, face à la nécessité de créer des cours du soir d'anglais à partir de 1937 (après l'échec de ces cours lors de tentatives antérieures), pour garder le monopole de l'excellence dans la ville, tout en transmettant la culture française, le consulat continue à assurer le collège de son soutien politique et financier ${ }^{36}$.

Les visites officielles de France (Haut-commissaire de Syrie, académiciens, professeurs d'universités françaises...) ainsi que les dons de livres et fournitures scolaires diverses, bourses, visas pour étudiants ${ }^{37}$ se multiplient.

Diplomatiquement, le consulat oriente les frères vers une coopération minimale avec les autorités mandataires, après leur refus initial. La stratégie progressivement adoptée consiste à introduire l'anglais et accepter les lois mandataires, comme moyen de diffusion de la culture française; la langue apparaît alors comme un refuge pour la culture.

La décision en vertu de laquelle les trois langues officielles de Palestine étaient reconnues exclusivement comme langue d'examens avait été prise après consultation du conseil d'enseignement, assemblée à laquelle les frères des écoles chrétiennes, bien que régulièrement invitées, ont toujours refusé de prendre part [...] Les frères imitant en cela l'attitude adoptée par la plupart des communautés françaises de Palestine [...] pensant défendre ainsi leur indépendance ${ }^{38}$.

Les frères seront heureux à l'avenir de collaborer avec le département de l'instruction publique, dont ils connaissent les bonnes dispositions à leur égard [...] Nous sommes en Palestine les doyens de l'enseignement et nous avons toujours travaillé pour le bien du pays. Nous ne demandons qu'à continuer ${ }^{39}$. 


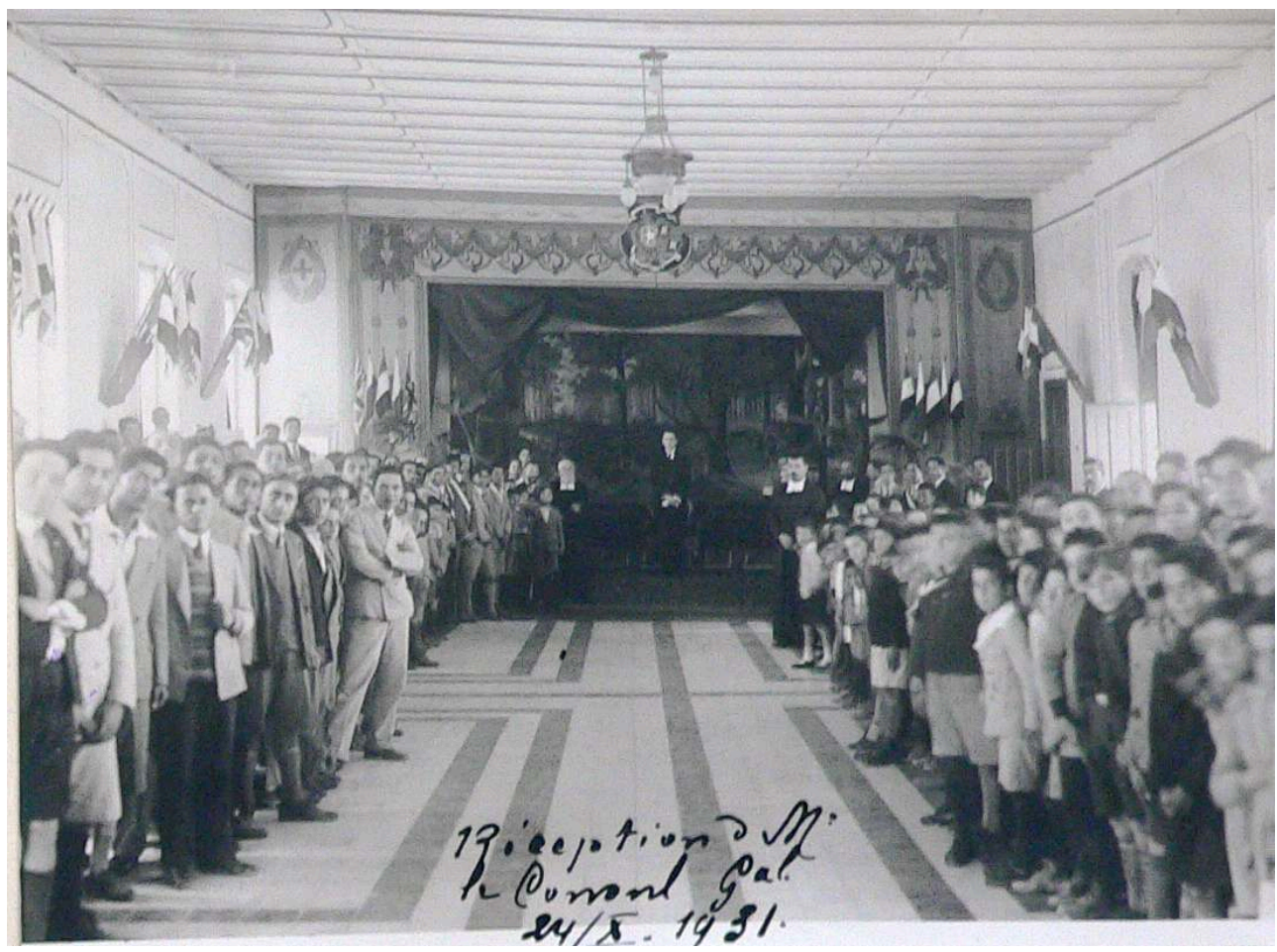

21 Le soutien global au collège est un aspect de la tentative de maintien de l'influence française, qui se joue aussi spatialement, avec la construction du nouveau consulat en $1929^{40}$, construit au même moment que la résidence du Haut-commissaire britannique.

\section{Concurrence éducative et religieuse}

Le contexte de concurrence dans lequel s'inscrivent les différentes tentatives des frères est marqué par la division entre pouvoirs chrétiens mais également par des pressions sur le collège de la part de la Propaganda Fide et du patriarcat latin. Plusieurs courriers reprochent aux frères leur patriotisme et leur volonté de préserver l'usage du français. Dans une lettre de 1921, Monseigneur Barlassina, patriarche de Jérusalem, invite les frères à ne pas se montrer aussi patriotiques :

les missionnaires doivent se garder d'insister sur l'enseignement de la langue de leur pays, pour ne pas paraître plus préoccupés des intérêts de leur patrie que du salut des âmes ${ }^{41}$.

La Propaganda Fide émet la même injonction : "les missionnaires ne doivent pas se soucier des choses temporelles [...] ils éviteront de chercher à propager leur langue maternelle à l'étranger ». Face au phénomène progressif de l'arabisation, le patriarcat invite les frères à améliorer leur enseignement de l'arabe et dispenser les cours de catéchisme uniquement en arabe.

Certains élèves partent du collège des Frères pour aller au collège de Terra Sancta, créé par les franciscains (qui accueille beaucoup moins d'élèves que les frères, et ne comporte pas d'école gratuite), dont un des buts est de rassembler une élite davantage anglophone et arabophone. Ceux-ci sont soutenus par de nombreux crédits du gouvernement italien (les rivalités inter-catholiques persistent durant la période mandataire) et du Vatican ${ }^{42}$ : achat d'un terrain quatre fois plus cher que celui des 
frères, création d'un magazine, et en 1929, concurrence plus ouverte encore quand l'italien est remplacé par le français au sein du cursus. Cependant, le collège conserve un nombre plus ou moins constant d'élèves sur la période. Parallèlement, les Italiens sont actifs durant cette période, au niveau de l'enseignement ${ }^{43}$.

\section{Savoirs, sociabilités et langue élitaires... de la fabrique des processus identitaires ${ }^{44}$}

La communauté française des frères habite dans la vieille ville et conserve une image de professeurs proches de la population locale. Durant cette période de crispations politiques et identitaires, une différenciation plus nette apparaît entre les élites du collège et l'école gratuite. Au sein du collège, la langue française rassemble autour de savoirs dispensés, de valeurs partagées et d'espaces de sociabilités ${ }^{45}$. Le collège agit donc comme une «antichambre de la position sociale $»^{46}$, et ce, avant le début du mandat britannique :

Les administrations, les banques et les maisons de commerce accueillent avec faveur les élèves diplômés. L'étude des langues arabe et anglaise est obligatoire au collège; celle de la langue turque est facultative [...] les deux écoles sont fréquentées par un grand nombre de musulmans ${ }^{47}$.

\section{Élites palestiniennes du collège, composantes et reconnaissance}

Troisième classe, 1928-1929 (archives du collège)

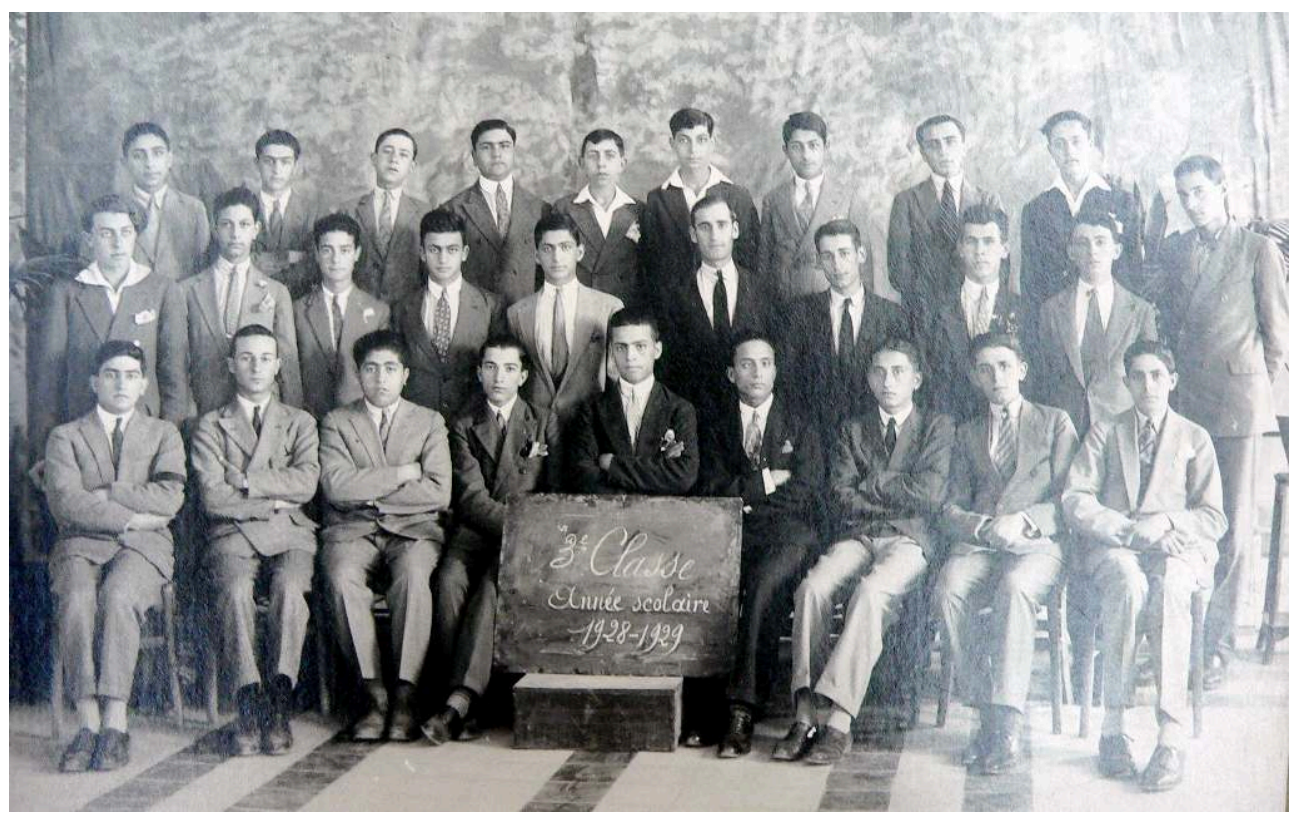

Ces élites sont plurielles, ethniquement et religieusement ${ }^{48}$. La majorité d'entre elles sont catholiques sur l'ensemble de la période ( $50 \%$ à $55 \%$ des élèves du collège), de nombreuses communautés chrétiennes sont présentes, ainsi que des musulmans et des juifs (de 8 à $11 \%$ des élèves, même période), mais peu d'Européens (maximum une vingtaine sur un total de 200 à 250 élèves du collège ; aucun en école gratuite) ${ }^{49}$. 
Les frères se singularisent auprès de l'ensemble de ces communautés par leur enseignement des langues et leur enseignement professionnel (augmentation sensible de $20 \%$ de plus de diplômes de comptabilité, brevets et certificats). Le curriculum, varié, est apprécié des parents, qui déplorent cependant le manque de formation de certains enseignants laïcs. Il comprend sur l'ensemble de la période : français, arabe, anglais, histoire, géographie, dessin, musique, gymnastique, mathématiques (arithmétique, algèbre, géométrie), sciences (physique, chimie, sciences naturelles). Pour l'enseignement professionnel: dactylographie, comptabilité. Il sera complété à partir de 1937 par des cours de sténographie. Tout au long de la période, le français reste la langue d'enseignement de référence, mais l'anglais et l'arabe augmentent sensiblement dans les emplois du temps des élèves. L'enjeu de l'enseignement dispensé par les frères est l'insertion professionnelle des élèves, ainsi que l'adaptation de leur cursus à la culture locale ${ }^{50}$. Même pendant les vacances d'été, un encadrement et des devoirs sont proposés aux parents. Les annales rapportent les attentes des parents ${ }^{51}$. Vers la fin de la période considérée, on note un nombre important de diplômés :

Fin juin 38, départ du frère François Louis. Les supérieurs l'ont autorisé à se rendre

à Londres pour y passer ses vacances afin de se perfectionner dans la langue

anglaise.

12 certificats en langue arabe

17 certificats d'enseignement secondaire français

23 diplômes de dactylographie

23 brevets d'études comptables

16 certificats de comptables ${ }^{52}$.

Les remises de diplômes sont nombreuses (diplômes mensuels, trimestriels, annuels, diplômes des devoirs de vacances), et lors de chacune de ces cérémonies, l'importance de l'éducation "à la française » est soulignée. Les frères garantissent une insertion importante de leurs élèves parmi les cadres supérieurs de la société palestinienne (7/10 des élèves ont un haut poste dans l'administration ${ }^{53}$ ). Certains membres du jury appartiennent aux élites elles-mêmes (cadres des banques notamment), et valident en partie les diplômes professionnels.

Cependant aucun centre d'enseignement supérieur n'apparaissant en Palestine sous le mandat, hormis la création de l'université hébraïque en 1925, qui s'adresse à un public différent, certains élèves (une minorité) partent à Beyrouth (université francophone Saint-Joseph ; à la fin de la période à l'American University, en raison de l'enseignement accru de l'anglais et du niveau plus faible des élèves en français) ou en Égypte (Institut de comptabilité supérieur des frères). Face aux événements de 1938, certaines familles commencent à émigrer au Liban ${ }^{54}$. D'autre part, face à la montée du nationalisme palestinien, la langue se charge davantage d'affect: les parents revendiquent un apprentissage plus important de l'arabe et de l'anglais tout en désirant le maintien d'une éducation à la française ${ }^{55}$.

\section{Sociabilités élitaires}

Quelques années après la fondation du collège, un club sportif et une chorale sont créés ; leurs activités augmentent par la suite, de l'avis des frères, contre le laxisme de la société ambiante ${ }^{56}$. Elles reflètent l'importance des pratiques culturelles liées à des pratiques linguistiques ${ }^{57}$. Les rencontres annuelles inter-collèges des frères favorisent la connaissance mutuelle de ces élites. 
31 D'autres associations, à vocation plus religieuse, proposent aussi des activités culturelles en français, et contribuent à fédérer les élites francophones. Il s'agit de l'Association du Sacré-Cœur (1906), de la Confrérie du Très Saint Enfant Christ (1909) élevée au rang d'Archiconfrérie en 1910 et reconnue par la papauté. En 1926, l'Amicale des anciens ${ }^{58}$ est constituée. Selon des témoignages oraux, des groupes de dialogues inter-religieux auraient été constitués durant les années 1930, consécutifs aux problèmes politiques affectant la vieille ville.

À partir de 1931, le collège compte 61 «non-chrétiens », dont 42 musulmans, par la suite ce chiffre ne cesse de s'accroître. Les élèves orthodoxes et protestants, parfois appelés "schismatiques ${ }^{59}$, constituent entre 30 et $35 \%$ des élèves. Excepté quelques périodes difficiles, comme les émeutes du mur des lamentations en 1928, ou la grande grève de protestation contre les Britanniques de 1936, qui touchent la fréquentation du collège aux printemps 1936 et $1938^{60}$, et qui en éloignent la plupart des élèves juifs, le collège accueille toutes ces communautés de manière constante durant cette période ${ }^{61}$, mais les tensions augmentent avant 1939 :

Révérend directeur, après avoir appris que votre Collège compte des élèves arabes et des élèves juifs [...] nous sommes arrivés aux conclusions suivantes [...] :

1. Puisque notre fin et le but que nous poursuivons est d'arriver à la liberté, à l'indépendance de notre pays, nous désirons beaucoup l'éducation de notre jeunesse.

2. Nous sommes arabes avant d'être musulmans ou chrétiens. C'est pourquoi tous ceux qui habitent notre pays doivent respecter notre patriotisme et nous respecter...

3. Nous voulons que notre jeunesse soit patriote avant d'être philosophe et savante.

4. Le mélange de notre jeunesse avec les élèves juifs affaiblit son patriotisme et produit un effet contraire à ce que nous avons mentionné ci-dessus.

C'est pour ces raisons importantes que nous vous prions d'expulser les élèves juifs de votre collège et de leur défendre d'avoir des rapports avec nos enfants en employant pour cela le moyen qui vous convient ${ }^{62}$.

L'heure n'est plus alors au patriotisme français... Le français reste la langue véhiculaire et la langue d'apprentissage au collège, mais cohabite de plus en plus avec l'anglais (langue officielle dans la société mais non véhiculaire au collège avant les années 1940) et l'arabe (également vernaculaire au collège). Le français est devenu une langue « dominée » dans la société.

\section{Le français, une langue des minorités devenue minoritaire?}

«Cette langue peut subir une éclipse, mais jamais une éclipse totale.» P. Dhorme, prieur des dominicains de Jérusalem, rapport sur la langue française, 1922 (AMAE, O 172)

Le collège des Frères s'ouvre progressivement aux classes moyennes durant le mandat britannique, peut-être la période voit-elle apparaître ce que l'on qualifiera après 1948 de « choix de la réussite individuelle». De par la qualité de son enseignement, de son corps enseignant, les cours professionnels proposés, même durant des périodes marquées par les difficultés, le collège s'assure un recrutement constant des élites francophones de Jérusalem, parallèlement à l'ouverture d'autres institutions visant ce même public. 
35 linguistique et culturelle française en Palestine : une politique culturelle qui se confond par certains aspects avec la politique religieuse, les frères étant les représentants de longue date de la France en Palestine. Cependant, face à la déconsidération de la langue française par les autorités mandataires britanniques, le consulat général encourage les frères à progressivement diffuser non plus seulement une image chrétienne de la France mais une image qui se veut un peu plus universaliste.

En 1939, l'anglais est la seule langue officielle et coexiste avec le français comme langue des élites, mais avant leur départ en 1948, les Britanniques restent peu reconnus pour leur rôle éducatif, tandis que le collège, perturbé dans son recrutement et son fonctionnement par les événements politiques, demeure un des collèges de référence pour les élites chrétiennes, aux côtés des élites anglophones récemment promues par le mandat. La langue et l'éducation françaises semblent devenir au début des années 1940, un " capital culturel thésaurisé ", voire victimes d'une " tentation de défection » ${ }^{63}$ de la part des élites, contrairement à l'avis du Consul général de France à Jérusalem.

On peut se demander si la France pourra maintenir sa position privilégiée en Orient en Terre Sainte. Cette position s'était affirmée entre 1914 et 1939 [...] Les anciens clients et amis du Proche orient ne se détourneront-ils pas d'elle, pour écouter la voix d'autres puissances, plus riches? De toutes façons, il reste là-bas une élite qui pense, et qui estime que, pour penser clairement, rien ne vaut les disciplines françaises. Aussi doit-on envisager l'avenir en se répétant ce proverbe arabe, si plein de foi dans la puissance du temps : « quand tu marches vers le soleil, l'ombre est derrière toi ! ${ }^{64}$

\section{BIBLIOGRAPHIE}

AUDISIO, Gabriel (dir.) (1998), Religion et identité, actes du colloque d'Aix-en-Provence, 24-26 octobre 1996, organisé par le CNRS et l'université de Provence, Publications de l'université de Provence.

BOURDIEU, Pierre (1982), Ce que parler veut dire, Fayard.

BOWMAN, H. E. (1942), Middle East Window, Longman.

BURROWS, M., « Mission civilisatrice, French Cultural Policy in the Middle East, 1860-1914 », The Historical Journal, 29, I, 1986, p. 109-135.

CABANEL, Patrick (2006), Une France en Méditerranée. Écoles, langue et culture françaises XIXe ${ }^{e} X^{e}$ siècles, Créaphis.

CABANEL, Patrick (1998), « Les deux vocations : catholique ou laïque ? La France, le Levant et l'Islam, 1860-1920 », in Religion et identité, Gabriel Audisio (dir.), Publications de l'université de Provence.

CALVET, Louis-Jean (1974), Linguistique et colonialisme : petit traité de glottophagie, Payot.

CARNOY, M. (1974), Education as cultural imperialism, Longman.

D’AUMALE, Jacques (1945), Voix de l'Orient, souvenirs d'un diplomate,Montréal, Variétés. 
HAJJAR, Joseph (1979), Le Vatican, la France et le catholicisme oriental (1878-1914), Beauchesne.

HUSSEINI SHAHID, Sirine (2005), Souvenirs de Jérusalem, Fayard.

ICHILOV, Orit \& MAZAWI, André Elias (1996), Between State and Church : Life - History of a FrenchCatholic School in Jaffa, Plenum Press.

KHALIDI, Rashid (1997), Palestinian identity, the construction of a modern national consciousness, Columbia University Press.

LAURENS, Henry (2002), La question de Palestine, tome 2, 1922-1947, une mission sacrée de civilisation, Fayard.

LAURENS, Henry (2002), Le retour des exilés, la lutte pour la Palestine, 1869-1997, Laffont.

LAURENS, Henry (2004), Orientales III, parcours et situations, CNRS Éditions.

MÉOUCHY, Nadine \& SLUGLETT, Peter (2004), Les mandats français et anglais dans une perspective comparative, Leyde, Brill.

NICAULT, Catherine (1999), Jérusalem 1850-1948, des Ottomans aux Anglais, entre coexistence spirituelle et déchirure politique, Paris, Autrement.

PIERRACCINI, Paolo (2006), « Un'Università cattolica a Gerusalemme ? L’Opera del cardinal Ferrari e il collegio francescano Di Terra Santa », Antonianum.

SEGUEV, Tom (2001), One Palestine, complete, Little Brown.

SHERMAN (2002), Mandate Days, Johns Hopkins.

TIBAWI, Abdul Latif (1956), Arab Education in Mandatory Palestine : a story of three decades of British administration, Luzac.

TRIMBUR, Dominique (2002), «L'ambition culturelle de la France en Palestine dans l'entre deux guerres ", in Entre rayonnement et réciprocité. Contributions à l'histoire de la diplomatie culturelle,Publications de la Sorbonne, p. 41-72.

VERMEREN, Pierre (2002), École, élite et pouvoir, Alizés.

\section{NOTES}

1. Rapport au ministère des Affaires étrangères sur la langue française, 1922, Service des CEuvres françaises (AMAE, SOFE par la suite), 0172.

2. Annuaire de l'œuuvre d'Orient, Bulletin de 1919.

3. Ce concept est davantage explicité par la suite. L'établissement propose également, dans une autre section, un enseignement primaire gratuit aux habitants catholiques du quartier chrétien de la vieille ville pour la grande majorité.

4. Paradoxalement, les archives étant restées longtemps inexploitées, parfois inaccessibles ou perdues, ce collège de référence est celui qui a suscité le moins d'études. Outre les archives britanniques (Chief Secretary Office files Records 1926-1948, E- Education and Social Welfare), certains documents qui permettent d'établir la vie quotidienne du collège sont épars, certains dans les institutions locales, d'autres ont été retrouvés dans les fonds personnels du collège. Enfin d'autres archives de l'Église permettent de compléter ce tableau ; il s'agit notamment de celles du Patriarcat latin, de la Custodie de Terre sainte, de la Maison Généralice des frères des écoles chrétiennes, du Vatican, de la Propaganda Fide ainsi que d'entretiens avec les anciens élèves (projet d'histoire orale).

5. Consul général de France à Jérusalem, 1931, Annales du collège. 
6. En ce qui concerne la société mandataire, le débat historiographique autour d'une définition précise du terme élite n'a pas cessé ( $c f$. notamment Hasan Kidr, Salim Tamari, Philipp Khoury), précisément pour les années 1930. Certains courants, issus de la conception américaine, rejettent tout statut particulier à l'élite. Différentes écoles, issues de la sociologie marxiste, définissent les élites comme ayant une place centrale dans la gestion et la défense des intérêts des classes dominantes. On considérera ici la conception véhiculée par l'expression présente dans la culture arabe classique Ahl al-hall wa-l-'aqd« ceux qui font et défont les choses, les dirigeants ». Plusieurs documents fournissent des listes d'élèves et leurs fonctions après l'obtention de leurs diplômes, dans la société palestinienne, notamment un document de 1947, citant la liste des participants pour l'accueil du frère supérieur général Athanase (élèves au collège durant la période mandataire), et certains prospectus de remise annuelle de diplômes. On remarque que cette élite formée chez les frères travaille essentiellement dans les banques et les administrations.

7. Durant cette période, Jérusalem obtient progressivement un statut de capitale politique ; une nouvelle classe moyenne est apparue à la fin $\mathrm{du} \mathrm{XIX}^{\mathrm{e}}$ siècle, constituée des membres des professions libérales (artisans, commerçants, intellectuels, fonctionnaires, employés de services ajoutés aux couches sociales traditionnelles, comme les grands propriétaires fonciers, les notables, et les hauts fonctionnaires de l'administration).

8. Henry Laurens, Orientales III, "Le levant arabe à la fin de l'époque ottomane, 1880-1914», CNRS, 2004. MAE, P. Dhorme, prieur de l'École biblique et archéologique de Jérusalem, La langue française en Palestine, AMAE, SOFE, O 172, 18 mai 1922. "Bien que la langue officielle de l'administration était le turc et la langue des natifs l'arabe, le français langue des autorités, pour ceux qui appartiennent à la société éduquée et pour communiquer avec les étrangers, après la langue maternelle, le plus important était le français ». Le collège de Jérusalem initie le mouvement d'expansion des frères à toute la Palestine mandataire en l'espace de dix ans. Le présent article ne répondra pas à la problématique du «modèle ", moins pertinente dans le cas d'un établissement missionnaire que dans le cas d'un établissement créé par les pouvoirs publics français avec pour vocation de répandre un modèle éducatif (par exemple le lycée de Galatasaray à Istanbul, $c f$. la thèse de doctorat de Gülsün Güvenli soutenue à l'EHESS en janvier 2007, Le lycée de Galatasaray (1868-1923). Histoire sociologique d'une institution scolaire.

9. Généralement abordés par le biais des champs diplomatique, politique, ou récemment économique,le mandat anglais - et l'influence du mandat français - ont rarement été abordés sous l'angle de la situation linguistique, révélatrice de tensions politiques et culturelles. Notamment : Méouchy, Nadine et Sluglett, Peter, Les mandats français et anglais dans une perspective comparative, Leyde, 2004 ; Tibawi, Abdul Latif, Arab Education in Mandatory Palestine : a story of three decades of British administration, Luzac, 1956.

10. On remarque qu'il s'agit de différents types d'expressions et usages de langue, qui s'interpénètrent en permanence au sein du collège : langue des cours, des activités de sociabilité, de communication, administrative, spirituelle, symbole de coexistence et de syncrétisme culturel. De même, les minorités considérées sont diverses : linguistiques, religieuses (les noncatholiques au sein de la population chrétienne, les juifs, les musulmans, au sein de l'institution deux catégories parallèlement majoritaires dans la population), ethniques.

11. Les schémas élaborés à partir de données des archives britanniques ont une légende en anglais dans l'article.

12. Nicault, Catherine, Jérusalem 1850-1948, des Ottomans aux Anglais, entre coexistence spirituelle et déchirure politique, Paris, Autrement, 1999, p. 103.

13. « 1914, 14 janvier : mort du frère Evagre, ce vieillard a été l'une des plus grandes et des plus sympathiques des figures françaises en orient [...] Véritable pionnier de la civilisation, le frère Évagre restera l'un des plus grands propagateurs de l'influence et de la langue françaises en Palestine et en Syrie [...] sa mort a été un deuil pour la ville de Jérusalem où il était connu et vénéré comme un père [...] 1914, dès le début de la guerre à Jérusalem, quand le bruit courut que 
notre collège allait être fermé, les notables musulmans, les députés, le cadi, le cheikh de la mosquée d'Omar, qui tous avaient leurs enfants chez nous, convoquèrent en assemblée les grandes familles de la ville et après délibération arrêtent qu'il fallait absolument s'opposer à la fermeture de notre établissement, cette école étant indispensable à la population [...]». Annales du collège, 1918-1919.

14. Progressivement, mais surtout à partir de 1936, le port de Jaffa et ses infrastructures économiques perdent de leur importance au profit de Caiffa et Jérusalem acquiert un poids politique en tant que capitale du mandat.

15. L'acte de langage est, dans le cadre de la Palestine mandataire, un cadre institutionnel qui s'inscrit dans une dialectique "dominant/dominé ", selon l'expression de P. Bourdieu. Les programmes culturels et linguistiques ont en effet des caractéristiques qui relèvent de "politiques linguistiques» (dans la définition des priorités et la continuité de l'action observable dans les différentes archives), même si le terme n'est guère utilisé à l'époque. $C f$. B. Laponce, Loi de Babel et autres régularités entre langue et politique, Presses de l'université Laval, 2006 et D. Ager, Language policy in France and Britain, the processes of policy,Londres, Cassel, 1996.

16. Institut archéologique allemand, notes attachées aux Annales du collège, 1905.

17. Pernot, M., Rapport sur un voyage d'étude à Constantinople, en Égypte et en Turquie d'Asie (janvieraoût 1912), Comité de défense des intérêts français au Proche-Orient, Firmin Didot, 1912 : «À voir avec quelle ardeur les sociétés allemandes, italiennes, anglaises, russes et américaines poursuivent en Orient leurs œuvres de pénétration méthodique et de lente conquête, et avec quel empressement les gouvernements étrangers encouragent le zèle des particuliers et les invitent à seconder par des entreprises privées leur action officielle, on comprend mieux la valeur des intérêts et de l'influence que nous possédons et que d'autres nous envient. »

18. Notamment tout ce qui concerne l'exemption des droits de douanes pour les établissements français, et leur inspection par le Haut-commissariat anglais.

19. Robert de Caix au ministre des Affaires étrangères, 28 octobre 1920, AMAE, E312/1, ${ }^{\circ} 404$.

20. Laurens, Henry, Orientales III, CNRS Éditions, 2004, p. 142.

21. P. Dhorme, La langue française en Palestine, AMAE, SOFE, 0 172, 18 mai 1922. Au début des années 1920 la France se pose en protectrice des catholiques et dans une moindre mesure des minoritaires, depuis la deuxième moitié du XIX". "Cette francophonisation tend à une francisation d'autant plus facile qu'elle est volontaire en dehors de tout système de domination ", Laurens, Henry, Orientales III, CNRS Éditions, 2004, p. 141.

22. AMAE, E 312/ 32, 26 avril 1924, « les langues d'examen [...] sont, à l'exclusion de toute autre, les trois langues officielles de la Palestine « anglais, arabe, hébreu » [...] cette décision [est] de nature à détourner les familles de nos maisons d'éducation où la langue d'enseignement est le français [M. Bowman, le ministre de l'Education Department en Palestine déclare au Consul général de Jérusalem que] dans l'avenir, les diplômes officiels donneraient seuls accès aux carrières officielles ou tout au moins que les candidats jouiraient d'un droit de préférence. »

23. Tibawi, Abdul Latif, Arab Education in Mandatory Palestine: a story of three decades of British administration, Luzac, 1956.

24. Annales du collège, 1919-1939.

25. Cette influence se décline d'une manière différente de celle du Liban, sous protectorat français. Les élites palestiniennes ont une plus grande conscience de leur identité linguistique, le français n'est pas perçu comme une deuxième langue maternelle par les parents des élèves ; il n'y a pas de cours du soir comme ceux proposés par Berlitzdans d'autres villes orientales.

26. Annales du collège, discours du Consul général lors de la remise des diplômes, 1931 ; AMAE, SOFE, O 139, Consulat général de France à Jérusalem, n 118, « Allocations Palestine - 1930 ».

27. Abbé Guerrin, Arras, visite à Jérusalem, 16 octobre 1920, rapport au MAE : « je n'ai pas à louer le dévouement et le patriotisme de nos établissements [...] je ne puis m'empêcher de me réjouir 
de l'union absolue et de la confiance réciproque que j'ai constatées entre toutes ces maisons et le représentant de la France ».

28. Rapport du frère Justinus sur les établissements des frères au Proche-Orient, 1919, Chambre de commerce et d'industrie de Marseille. Également Maurice Barrès, Enquête aux pays du Levant, tome 1, Paris, Plon, 1923, p. 193. Il est d'avis de donner «plus de poids aux actions françaises, 'plus spirituelles', qui doivent permettre de s'attacher le cœur des orientaux. Nous devons maintenir nos positions et par conséquent garder nos pièces maitresses, à savoir nos congrégations enseignantes et charitables ».

29. Lettre du frère Alexis François, procureur de l'Institut, au frère Allais Charles, de Rome le 16 mai 1924 (archives de la Maison Généralice) : la communauté des religieux craint que le frère visiteur de Jérusalem (qui détient une fonction d'inspecteur pédagogique et religieux pour la communauté) n'ait commis des imprudences de langage en parlant de la mission patriotique des frères sans citer leur apostolat religieux.

30. Annales du collège, 1927.

31. Trimbur, Dominique, "L'ambition culturelle de la France en Palestine dans l'entre deux guerres ", in Entre rayonnement et réciprocité. Contributions à l'histoire de la diplomatie culturelle, Publications de la Sorbonne, 2002, p. 41-72. Les changements de position par rapport au français et le public juif et les représentants sionistes sont aussi marqués par ce changement. La France sort du strict cadre de l'AIU pour prendre en compte d'autres représentants. Ces aspects ne sont pas explicités dans cette étude. Fabrizio, Daniela, La battaglia delle scuole in Palestina: tradizione e modernità nell'educazione giovanile ebraica, Franco Angeli, 2003.

32. « 13 et 14 mai 1926 : solennité du cinquantenaire du collège. Pendant 3 jours, des instructions furent données à Notre Dame de France et le 15, les bienfaiteurs, les amis et les anciens élèves, dans un banquet tout à fait intime, fraternisaient. Le Consul général voulut bien présider cette réunion de famille ». Annales du collège, 1926.

33. Discours du député Lecour Grandmaison, Bulletin de l'Euvre d'Orient, 1921.

34. Autres sources financières pour le collège : celles de l'œEuvre d'Orient, de la Propagation de la Foi, mais parfois ces organismes renvoient ouvertement au soutien attendu de la France.

35. «Je pense que chacune de nos œuvres doit être considérée comme la pierre d'un édifice que la France a lentement construit avec le dévouement de nos religieux et de nos religieuses ; c'est ce ciment qui le maintient encore debout et assez fièrement mais l'aide financière de l'État est nécessaire, le moment serait mal choisi pour la réduire.", AMAE, E 312/12, n 61, Extrait du rapport du Contre-amiral Joubert, commandant la division navale du Levant au sujet des établissements français de Palestine, le 9 décembre 1932.

36. «Pour ces jeunes gens qui forment une partie de notre clientèle, notre culture est un luxe qui ne suffit plus à leur assurer un avenir [...] [Ils devraient suivre] une formation en arabe et en anglais, tout en continuant à recevoir une éducation à la française ", AMAE, série B, 274, $n^{\circ} 24$, le ministre au Consul général de Jérusalem, 17 juillet 1934.

37. AMAE, SOFE, O 172, O 369.

38. AMAE, E 312, 32, 26 avril 1924.

39. Lettre du frère Polycarpe, directeur, à M. Bowman, 1925.

40. "Les travaux commencèrent à la fin de 1929, quand les troubles qui venaient d'avoir lieu se furent un peu calmés [...] le bâtiment construit par Favier est un des plus beaux immeubles consulaires possédés par la France [...] Devant la maison, Favier avait disposé un miroir d'eau, ornement de grand luxe en Palestine, et une pergola. Le consulat de France devint un centre d'art français et il n'était touriste de marque qui ne tint à le visiter. Gloire suprême: les Italiens désireux de nous imiter, envoyèrent un de leurs architectes pour s'en inspirer. Mais finalement, ils ne construisirent rien ». V. d'Aumale, Consul général de Jérusalem, Voix d'Orient, souvenirs d'un diplomate,Montréal, Variétés, 1945, p. 167.

41. Circulaire de Mgr. Barlassina, patriarche de Jérusalem, 2 octobre 1920, Annales du collège. 
42. Lettre du frère Florin au frère assistant, du 15 novembre 1931, sur les franciscains et leur propagande en défaveur du collège des frères; des racoleurs auraient été postés aux portes d'entrée du collège, vantant les mérites d'une éducation meilleure et moins chère au collège de Terra Sancta.

43. À Jaffa et Caiffa la concurrence éducative est vive : les établissements ouverts par les Italiens sont souvent gratuits. À Jérusalem, le collège de Terra Sancta des franciscains s'appuie sur des actions associatives et sociales plus subventionnées que ne le sont celles du collège des Frères. La concurrence touche également le secteur médical (hôpital Saint Louis des Français notamment, nombreux orphelinats). Ichilov, Orit et Mazawi, André Elias, Between State and Church : Life-History of a French-Catholic School in Jaffa,Plenum Press, 1996.

44. Le terme d'«identification" paraît plus approprié que le terme réifiant d'identité, $c f . R$. Brubaker, "Au-delà de l'identité ", Actes de la recherche en sciences sociales,199, p. 66-85, 2001 ; Avanza et Laferté, « Dépasser la construction des identités », Genèses,61, p. 134-152, 2005.

45. Langlois, E., 1931, « Rapport sur l'enseignement donné par les frères des écoles chrétiennes, spécialement dans leur collège du Proche-Orient », Bulletin des écoles chrétiennes, 1931, 327-339.

46. L'expression est de J. Bocquet, Le collège Saint-Vincent des pères lazaristes de Damas: l'enseignement français en Syrie (1864-1967), thèse de doctorat, 2002.

47. Pernot, M., Rapport sur un voyage d'étude à Constantinople, en Égypte et en Turquie d'Asie (janvieraoût 1912), Comité de défense des intérêts français au Proche-Orient, Firmin Didot, 1912, p. 97.

48. Il s'agit de minorités ethniques (les Grecs), religieuses et linguistiques (les juifs), religieuses linguistiques et ethniques (les Arméniens orthodoxes sont les plus nombreux au collège).

49. Données recueillies dans les Blue Book, Education Department, Annual Reports; statistiques fournies au consulat chaque année, Annales du collège et MAE, SOFE.

50. En 1912 déjà, le rapport de M. Pernot, insistait sur la nécessité, pour les frères et autres établissements de référence de la Palestine ottomane d'un «enseignement pratique et professionnel », « la culture générale d'un oriental, ne peut être identique à celle d'un Français et il ne servirait de rien qu'elle le fut [...]; [un] enseignement des langues indigènes, organisé suivant nos méthodes, mais fondé sur leur culture nationale et répondant à leurs besoins [...] serait la preuve la meilleure et la plus opportune du désintéressement et de la tolérance dont leur œuvre est inspirée ", ibid., p. 283.

51. Annales du collège, «peur de l'oisiveté, mauvaises fréquentations [...] bien choisir ses amis, toujours de haute qualité ».

52. Annales du collège, 1938 (sur un total de plus de 240 élèves; quelques divergences sur le nombre d'inscrits entre les sources du Blue Book annuel britannique et les Annales). Cependant, durant les années 1930, à la suite de problèmes survenus à des étudiants voulant s'inscrire en faculté de médecine et n'ayant pas obtenu d'équivalence pour leur baccalauréat, le consulat général reconnaît, ainsi que les frères, que les diplômes du collège ne sont pas équivalents aux diplômes français (selon le bureau des Euvres françaises, il y a un à deux ans de décalage). AMAE, Nantes, 0 172, 0372.

53. 1933, Rapport du frère visiteur (archives de la Maison Généralice).

54. Les frères se plaignent du départ de certains de leurs meilleurs élèves, Annales, 1939.

55. Le collège des frères reste en retrait des revendications identitaires, des vastes mouvements de grèves, contrairement au collège gouvernemental (le Arab College) notamment. Après la loi de 1924 sur l'annulation du français comme langue d'examen, les frères renforcent progressivement l'apprentissage de l'anglais et de manière très significative après le milieu des années 1930.

56. Pour la période, il s'agit essentiellement du développement du YMCA, Young Men Christian Association.

57. Mouktatal al dourousse est l'une des rares associations de cours de langues qui comprend 40 musulmans et chrétiens. Elle réunit ses membres durant quelques années autour de petites conférences et des cours de langue française. Hymne à l'occasion du 14 juillet devant le Consul 
général, « œuvres françaises, bureau des vertus et des sciences [...] vous avez été pour la nation arabe une mère compatissante !». Comparativement à la scène culturelle libanaise, surtout celle de Beyrouth, Jérusalem n'accueille pas autant de conférences et événements littéraires. L'instauration d'un Centre de Culture française dans la partie ouest de Jérusalem durant les années 1930 vise un public davantage juif, MAE Nantes, 0369.

58. Bulletin de l'Institut des frères, 1925, p. 90-91 (réunion d'anciens élèves).

59. Rapport du frère visiteur en 1930, sur 1929 «le collège a bonne réputation même parmi les schismatiques » (archives de la Maison Généralice).

60. 1928-1929 : incidents et émeutes près du mur des lamentations, fortes tensions, $c f$. le rapport de la commission Shaw ; 1936-1938 : grève générale, cf. le rapport de la commission Peel ; «Les troubles de Palestine », frère Régis, Jaffa, le 10 octobre 1938 (archives de la Maison Généralice).

61. La plupart d'entre eux sont élèves du collège, par la réputation des diplômes et centralité des frères, plus rares à l'école gratuite où la plupart des élèves vont encore dans des écoles confessionnelles ou à l'école de l'AIU, Alliance israélite universelle, pour les élèves juifs.

62. Le général courageux du district de Ramallah et de l'occident du Jourdain, le cheikh Abd El fath Mohamad, 28 octobre 1938, AMAE, SOFE, 0372.

63. B. Laponce, Loi de Babel et autres régularités entre langue et politique, Presses de l'université Laval, 2006, p. 28.

64. V. d'Aumale, Voix d'Orient, ibid., p. 247.

\section{RÉSUMÉS}

L'article analyse la position du collège des Frères de Jérusalem, de 1922 (Jérusalem devient alors progressivement la capitale du mandat britannique) à 1939, période de concurrence éducative. Après avoir accueilli les élites ottomanes, le collège est alors le théâtre d'une nouvelle définition et formation des élites locales (le français joue encore un rôle important dans la formation des élites politiques et commerçantes jusqu'au milieu des années 1930). Souvent accusés d'être des missionnaires français trop patriotes par les autorités religieuses, les frères proposent un enseignement et une éducation chrétiens sans prosélytisme actif envers les différentes communautés religieuses. Soutenu par le consulat, il offre un pendant à l'influence anglaise et agit comme une plate-forme de l'influence culturelle française, en adaptant notamment son curriculum.

This article analyses the position of this French catholic school, from 1922 (Jerusalem becomes progressively the capital of the British Mandate), until 1939, period of educative concurrence. Elites' college under the Ottomans, the school proposes a new definition and formation to the local elites (French still plays an important role in the formation of the political and commercial elites until the mid 1930). Often accused of French patriotism by the religious authorities, the Brothers propose a non-proselyte Christian education to pupils from various religious communities. Supported by the French consulate, it offers a counterpart to the British influence and a platform for the French cultural influence, adapting especially its curriculum. 
INDEX

Mots-clés : politiques linguistiques, identités confessionnelles, Palestine mandataire britannique, Catholiques palestiniens, présence française en Palestine, Frères des écoles chrétiennes, Jérusalem, éducation missionnaire

Keywords : Linguistic policies, denominational identities, British mandate in Palestine, Catholics in Palestine, French presence in Palestine, Brothers of the Christian schools, Jerusalem, missionary education

\section{AUTEUR}

\section{KARÈNE SANCHEZ-SUMMERER}

Université de Leyde, Pays-Bas 